\title{
Two-dimensional gel electrophoresis image registration using block-matching techniques and deformation models
}

\author{
Alvaro Rodriguez ${ }^{\mathrm{a}}$, Carlos Fernandez-Lozano ${ }^{\mathrm{a}}$, Julian Dorado ${ }^{\mathrm{a}}$, Juan R. Rabuñal ${ }^{\mathrm{a}, \mathrm{b}}$ \\ ${ }^{a}$ Department of Information and Communications Technologies, University of A Coruña, Campus de Elviña, \\ 15071 A Coruña, Spain \\ ${ }^{b}$ Centre of Technological Innovation in Construction and Civil Engineering (CITEEC), University of A \\ Coruña, Campus de Elviña, 15071 A Coruña, Spain
}

\begin{abstract}
Block-matching techniques have been widely used in the task of estimating displacement in medical images, and they represent the best approach in scenes with deformable structures such as tissues, fluids, and gels. In this article, a new iterative block-matching technique - based on successive deformation, search, fitting, filtering, and interpolation stages-is proposed to measure elastic displacements in two-dimensional polyacrylamide gel electrophoresis (2D-PAGE) images. The proposed technique uses different deformation models in the task of correlating proteins in real 2D electrophoresis gel images, obtaining an accuracy of $96.6 \%$ and improving the results obtained with other techniques. This technique represents a general solution, being easy to adapt to different $2 \mathrm{D}$ deformable cases and providing an experimental reference for blockmatching algorithms.
\end{abstract}

Keywords

2D gel images; Block-matching; Deformations; Electrophoresis; Optical flow; Proteomics

Proteomics is the study of protein properties in a cell, tissue, or serum aimed at obtaining a global integrated view of disease, physiological and biochemical processes of cells, and regulatory networks. One of the most powerful techniques used to analyze protein mixtures extracted from cells, tissues, or other biological samples is two-dimensional polyacrylamide gel electrophoresis (2D-PAGE). ${ }^{1}$ With this method, proteins are separated by molecular weight and isoelectric point using a controlled laboratory process and digital imaging equipment [1].

2D-PAGE images represent 2D patterns of proteins derived from a sample that appear in the images as dark spots and where the sizes of the spots depend on the amounts of protein. In association studies, a pair of images is compared to find differences between proteins of interest. For this purpose, it is necessary to register the images and find the spots' correspondence.

The analysis of 2D gel images is a bottleneck in proteomics research due to potential displacements, appearance changes between proteins, or differences in protein expression and experimental conditions [2] and [3]. Over the years, several works studied this problem [4], [5] and [6], but the correspondence between proteins problem is still far from a standard solution.

In this work, a block-matching technique is proposed to be used as a pair of 2D electrophoresis gel images considering the test image as a deformation of the reference image so that the correspondence between the different proteins of the pair is estimated by analyzing the 
deformation. Block-matching techniques calculate the displacement of points $\left(x_{i}, y_{i}\right)$ defined in a source image $I$ by comparing the region or block around them with candidate blocks defined in a destination image $I^{\prime}$, representing each candidate block with a possible displacement of the original region, and selecting the block in $I^{\prime}$ centered at $\left(x_{i}{ }^{\prime}, y_{i}{ }^{\prime}\right)$ with the largest similarity value with the original one.

Over time, several works have focused on block-matching techniques, and important contributions were made. To increase accuracy, sub-pixel estimation using parabola fitting over three points was used in several works [7] and [8]. In addition, multiresolution approaches were widely proposed [9], [10] and [11]. A related contribution is the adaptive reduction of the search area [12] and [13]. Another important contribution was the analysis of displacements in the frequency domain using fast Fourier transforms (FFTs) to increase performance [8] and [14].

Based on early iterative image registration principles [15], some new methods have been proposed to explicitly include deformation in the search process [16]. In these techniques, dense fields are calculated iteratively by interpolating the block displacement field. Some examples of these techniques are based on the use of B-spline interpolation [17], first-order bidimensional Taylor series [16], and radial basis functions (RBFs) [9] and [18].

In addition, some works have used different approaches to improve the obtained flow. Some examples include the use of the particle swarm optimization technique [19], the inclusion of edge analysis [20], the use of feature information [21] and [22], and the use of Markov random fields (MRFs) [23].

The main advantages of block-matching techniques are simplicity, flexibility, robustness, and locality. However, these techniques are limited due to the block concept itself. Because a block has a size and shape defined a priori, this leads to wrong measurements near discontinuities in the motion field [24] and [25].

Several works have attempted, with some success, to reduce this problem by using a multilayer approach [26], pairwise affinities based on boundaries [24], adaptive shape windows [27], [28], [29], [30], [31] and [32], adaptive support weight windows [33], barycentric corrections [34], feature matching methods [35], and stereo analysis of cost volumes [25]. But the question is still far from having a standard solution.

Currently, it can be assumed that block matching might not be appropriate when analyzing a scene with different objects moving with different motions. However, it is one of the most robust methods for extracting the displacement field of a surface without reference points such as corners and edges. Therefore, block matching has been applied in several fields such as in analysis of flows [16] and deformable materials [36]. In medical imaging, its general application was studied in Refs. [37] and [38], and the use of block matching in rigid medical image registration was shown in Ref. [17] where a B-spline technique was visually tested with different examples of medical images. More concrete works have applied block matching to ultrasound images [39] and [40], echocardiography images [41], [42] and [43], and magnetic resonance imaging (MRI) brain images [44] and [45].

Current block-matching algorithms are created for each problem [17], usually using traditional noniterative and nondeformable techniques [36]. In this work, a general solution for the 2D scenario is provided. This approach has the advantage of being easily adaptable to different problems, allowing comparison with different motion algorithms. 
The main contributions of this article are as follows:

- A block-matching algorithm is proposed to solve the medical problem of matching proteins in images of 2D electrophoresis gels, defining a methodology to conduct and evaluate assays and improving the performance and accuracy of available state-of-the-art techniques.

- The proposed algorithm uses a multiresolution iterative process integrated with several deformation models that can be used as restrictions to guarantee the smoothness and freedom of the flow and to interpolate a dense flow for each pixel from an arbitrary set of points, allowing the registration of a warped image.

- A new methodology to conduct and evaluate experiments with 2D electrophoresis gels has been carried out.

\section{Materials and methods}

The 2D-PAGE image registration problem involves correlating the same protein in two 2D electrophoresis gel images from the same tissue but from different patients and obtained in different conditions (Fig. 1). The proposed technique was applied to the problem, assuming that one image can be mapped in the space of a reference image through a process of deformation.

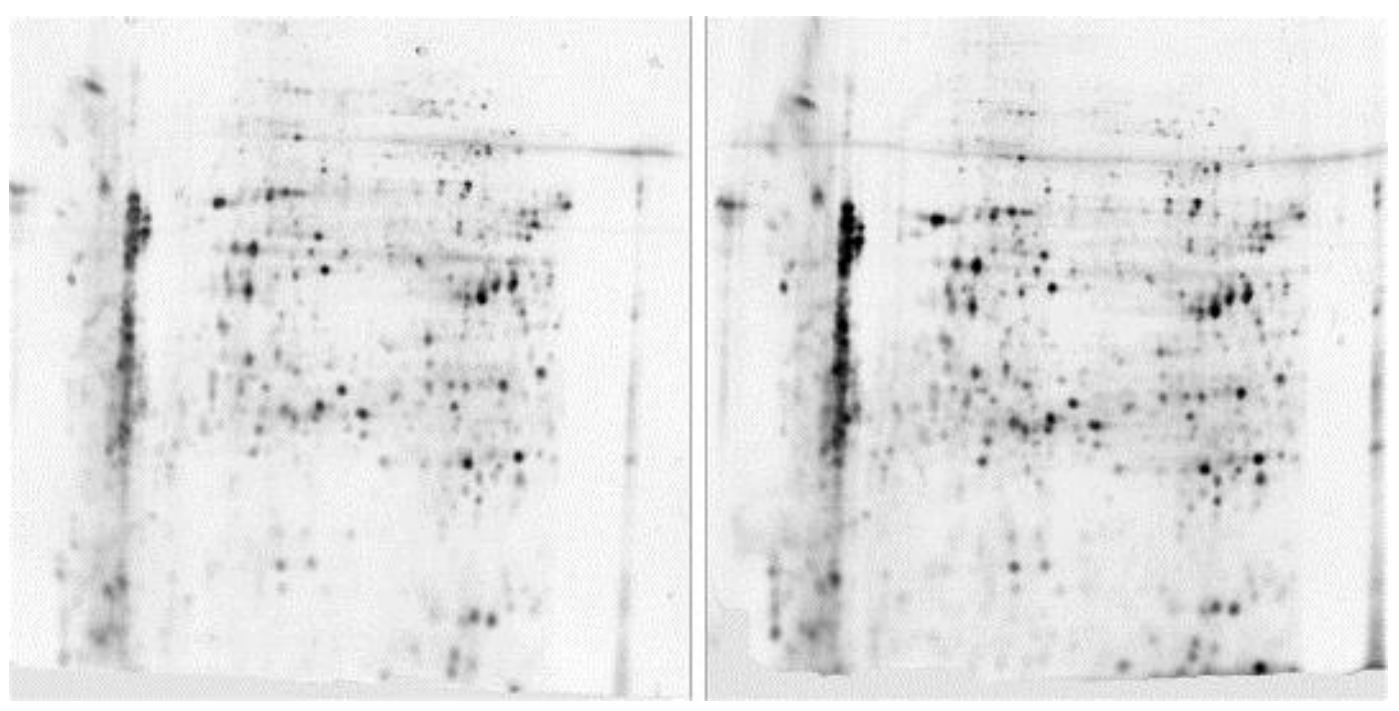

Fig.1. A pair of 2D electrophoresis silver-stained gel images that will be compared. Left: Reference image. Right: Test image.

Because there are no published datasets with ground truth data in this field, and a standard methodology to conduct experiments or evaluate protein-matching algorithms does not exist, eight samples (grouped into four pairs) of 2D gel electrophoresis images were selected from the dataset of Yang and coworkers [4]. These images have been used in several publications [46] and [47], and they correspond to an experiment where the effect of a plant extract on the protein expression of IBR3 human dermal fibroblasts was investigated. Both control and candidate samples were taken from homogeneous cell cultures grown in the laboratory where the candidate samples were treated with the plant extract. 
The conducted tests correspond to intergroup comparisons with $1024 \times 10248$-bit images, and $2 \mathrm{D}$ protein separation patterns were visualized by silver staining using standard protocols [48] and [49].

The defined methodology to evaluate the proposed registration technique on the dataset uses the following steps. First, the ground truth data are obtained from a pair of images by selecting proteins with Beads [50], a software program for spot detection in 2D gel images using surface information, and then correlating the detected spots using the criterion of experts. Then, the accuracy is measured for every point corresponding to a protein detected by Beads and taking into account only proteins expressed in the two images.

\section{Proposed technique}

The proposed technique divides the image into regular regions called blocks and solves the correspondence problem for each block (Fig. 2). To this end, it uses an iterative approach with different stages.

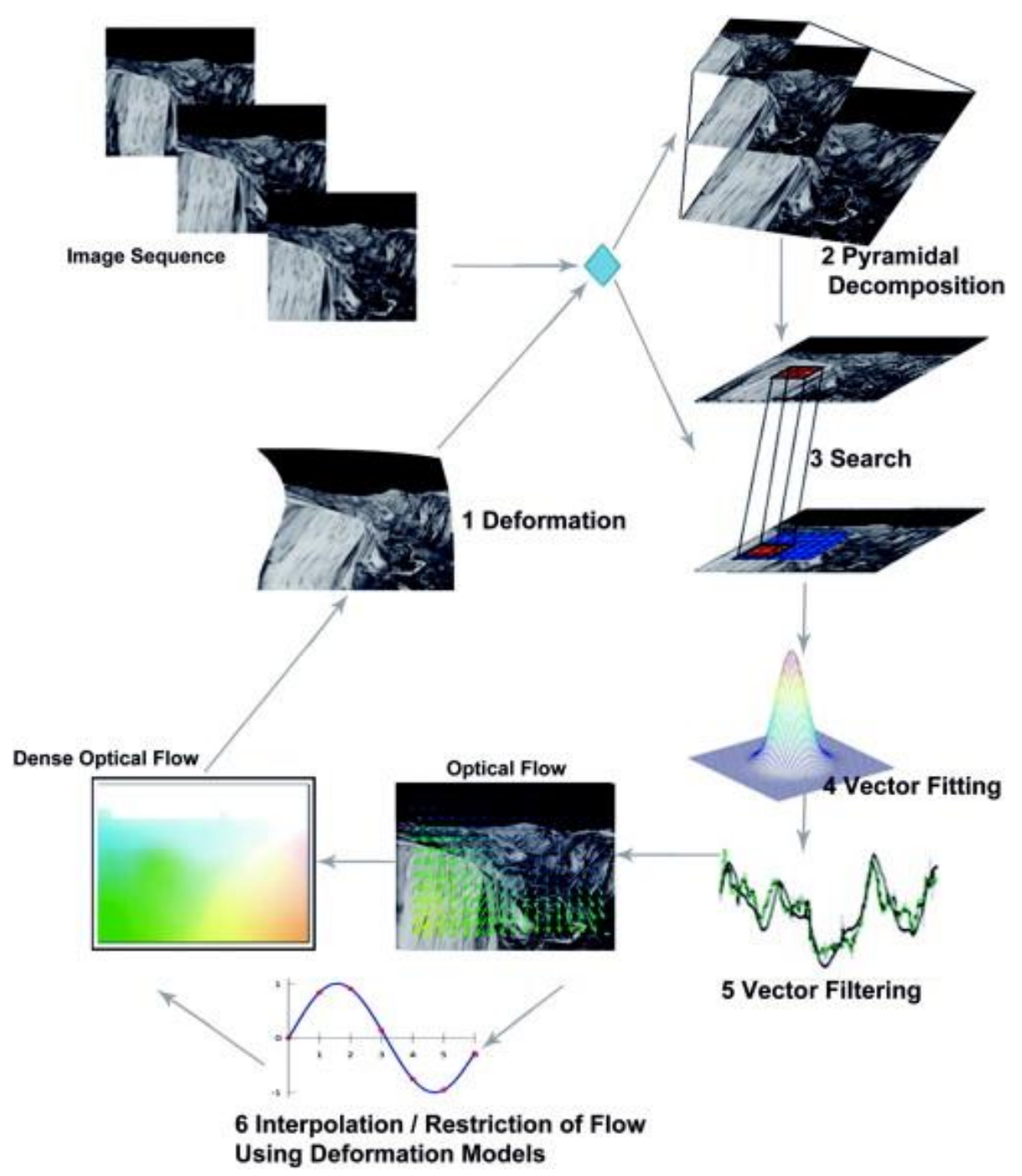

Fig.2. General scheme of the proposed algorithm. 
The main steps of the algorithm to analyse the displacement between two frames are as follows:

(1) The displacement field from the previous iteration is used to update the search area. Then, a deformation model is applied to change the shape of the block.

(2) Optionally, the image is down-sampled, whereas the block size remains constant and the search range is reduced. The number of pyramids can be selected by the user.

(3) A similarity metric and a search strategy are used to calculate the best displacement for each block.

(4) The obtained vectors are filtered and smoothed. Here, local root mean square (RMS) is used to remove outliers, and a bidimensional Gaussian filter is used to obtain a soft field.

(5) The similarity values are used to perform a fitting to a selected function to achieve a higher accuracy.

(6) The optical flow is processed with a deformation model used to interpolate the displacements and to impose restrictions on the displacement field. Then, an estimation of the deformed image is obtained from the dense field using a bilinear model to obtain nondiscrete image values. The deformed image is used in the next iteration to refine the results.

The block-matching model considers only linear displacements, making the assumption that if the time interval between the compared images and the regions or blocks are small enough [51], then the second-order effects may be disregarded, so body deformations may be calculated from the locally linear displacements in every region. Current block-matching techniques do not face this assumption properly, using several pyramidal decomposition stages or not addressing image sequences properly, measuring the total displacement in a sequence from the initial frame (augmenting the time interval).

The proposed technique uses the linear assumption only between consecutive frames, obtaining a linear estimation of the movement in the first iteration and computing deformations in subsequent iterations from previous ones. To this end, the source frame and the source block are dynamically updated after a number of images or when the displacement reaches a threshold. In addition, previous displacements are used to update the search area and the shape of the block even if the source image and source blocks are not updated, and the pyramidal decomposition is optional.

With this procedure, each measurement point follows the position of the original point through time, and movement can be calculated without violating the locality condition.

\section{Block-matching algorithm}

The proposed algorithm measures movements using the statistical similarity of the gray levels in each region of the image. For this purpose, a region or block is formally defined as a sub-area of the image of a particular and constant size and shape. Therefore, given a block $B_{x y}$, the similarity value $S_{u, v}$ will be the result of comparing the initial block with a candidate one $B_{x^{\prime} y^{\prime}}^{\prime}$ using a similarity function $f\left(B_{x y}, B_{x^{\prime} y^{\prime}}^{\prime}\right)$, which output measures the confidence in the hypothesis $B_{x y}=B_{x^{\prime} y^{\prime}}^{\prime}$ being $d(x, y)=(u, v)$, a displacement accomplishing $(x+u, y+x)=\left(x^{\prime}, y^{\prime}\right)$.

In the proposed technique, the Pearson correlation quotient $(R)$ has been used. It measures the strength of linear dependence between two variables. The Pearson correlation quotient has the advantage of being invariant to the average gray level: 


$$
R\left(B_{x y}, B_{x^{\prime} y^{\prime}}^{\prime}\right)=\frac{\sum_{x, y} \quad\left(\left(B_{x y}(p)-\mu\right) \times\left(B_{x^{\prime} y^{\prime}}^{\prime}(p)-\mu^{\prime}\right)\right.}{\sqrt{\sum_{p}\left(B_{x y}(p)-\mu\right)^{2} \times \sum_{p}\left(B_{x^{\prime} y^{\prime}}^{\prime}(p)-\mu^{\prime}\right)^{2}}}
$$

The data obtained in the similarity analysis led us to the most probable discrete displacement. In addition, to achieve measurements below the pixel level, these correlation values can be translated to a continuous space using a fitting technique.

In this work, 2D Gaussian and polynomial functions have been used with the LevenbergMarquardt (L-M) technique, which can be expressed as follows:

$\left(J_{n \times m}^{T} \times J_{n \times m}+d \times I_{m}\right) \times I n c_{m \times 1}=J_{n \times m}^{T} \times E_{n \times 1}$,

where $E_{n \times 1}$ is the error matrix calculated using $n$ observations and the values predicted with the model, $J_{n \times m}$ is the Jacobian matrix of the used function, $I_{m}$ is the identity matrix, $I n c_{m \times 1}$ is the vector of increments for the next iteration, and $d>0$ is a constant adjusted in each iteration $k$ according to the residual sum of squares (RSS) as shown in Eq. (3):

$$
\begin{gathered}
R S S^{k}<R S S^{k-1} \rightarrow d^{k+1}=d^{k} / 2^{k} \\
R S S^{k} \geqslant R S S^{k-1} \rightarrow d^{k+1}=d^{k} \times 2^{k} .
\end{gathered}
$$

An example of L-M fitting is illustrated in Fig. 3.
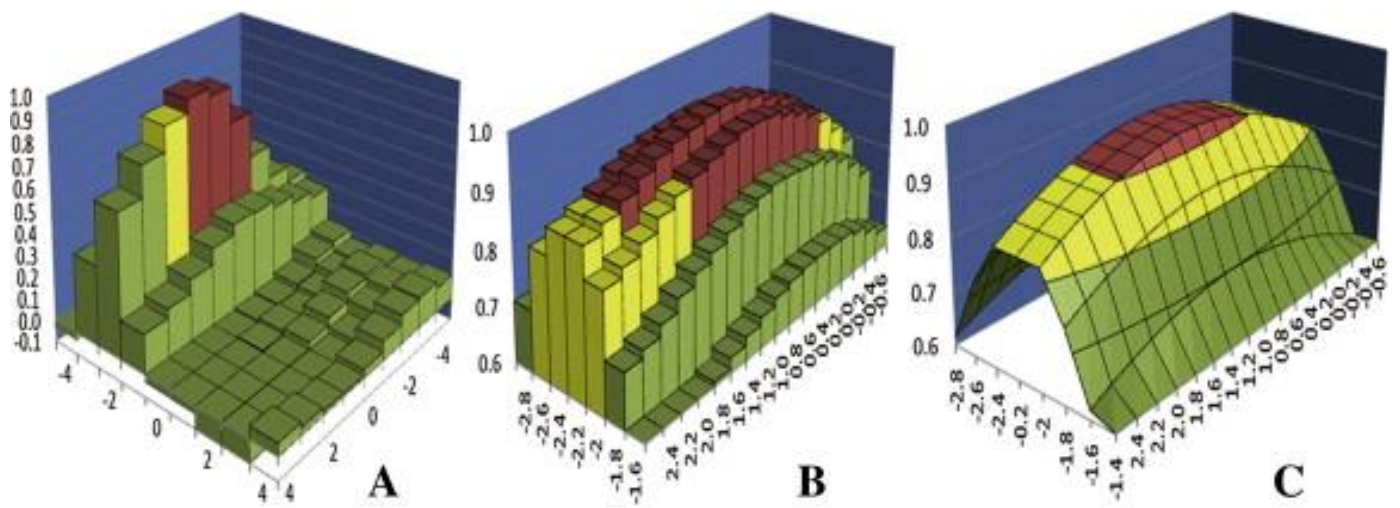

Fig.3. Similarity peak in a synthetic image sequence: (A) discrete correlation values; (B) correlation values near the peak using bilinear interpolation of the image; (C) L-M fitting performed with a Gaussian model using a $3 \times 3$ window of discrete values around the maximum.

\section{Deformation models}

A deformation model is a transformation that maps all positions in one image plane to positions in a second plane. Therefore, the deformation of an image is primarily a transformation of the plane to itself where the gray-level values are transformed according their associated coordinates.

In this case, the transformation of a set of points $\left(x_{i}, y_{i}\right)$, which has been calculated using the block-matching technique, is used to find a transformation model for each pixel of the image: 
$f: R^{2} \rightarrow R^{2} f(x, y)=\left(x^{\prime}, y^{\prime}\right)$,

where the function $f$ must satisfy the conditions $f\left(x_{i}, y_{i}\right)=\left(x_{i}{ }^{\prime}, y_{i}{ }^{\prime}\right)$ for the known points.

It should be noted that the terms interpolation and warping are, at this point, equivalent concepts. Furthermore, they are also related to filtering and smoothing because the warping model will be a compromise between a smooth distortion and one that achieves a good match.

\section{Parametric models}

Parametric deformation models are easy to compute and need only a few points to be solved. They model simple transformations such as scaling, rotation, and linear displacement. They are useful in many applications where it is important to use a transformation that is no more general than it need be [52].

In the proposed algorithm, the parametric models can be used as a constraint in the first iterations of the algorithm to remove outliers and the linear component of the deformation. Then, the search area can be reduced in the next iterations where more general models can also be used [52].

The mathematical formulation of the models used in this article can be expressed as described here. Linear displacement is the traditional model of block matching. Its mathematical formulation can be expressed as follows:

Linear Displacement $\left\{\begin{array}{l}x^{\prime}=x+a \\ y^{\prime}=y+b\end{array}\right.$.

The affine transformation is a six-parameter transformation. It models changes in the shape, preserving the collinearity relation between points.

This transformation is modeled with first-order polynomial equations as follows:

Affine $\left\{\begin{array}{l}x^{\prime}=a_{0}+a_{1} \times x+a_{2} \times y \\ y^{\prime}=b_{0}+b_{1} \times x+b_{2} \times y\end{array}\right.$.

The homography transformation, or perspective transformation, is an eight-parameter nonlinear transformation. It models the projection of a planar object viewed from a point in space. Their equations can be expressed as follows:

Homography $\left\{\begin{array}{l}x^{\prime}=\left(a_{0}+a_{1} \times x+a_{2} \times y\right) /\left(c_{0} \times x+c_{1} \times y+1\right) \\ y^{\prime}=\left(b_{0}+b_{1} \times x+b_{2} \times y\right) /\left(c_{0} \times x+c_{1} \times y+1\right)\end{array}\right.$.

\section{Radial basis function models}

Radial functions have proven to be an effective tool in multivariate interpolation problems of scattered data [53]. These techniques solve the interpolation warping problem using a set of radial functions $S$ defined in a set of points $\left(x_{i}, y_{i}\right)$ as follows:

$f: R^{2} \rightarrow R^{2} f(x, y)=\left(x^{\prime}, y^{\prime}\right)$
$f(x, y)=\sum_{i} c_{i} S\left(\left\|x-x_{i}, y-y_{i}\right\|\right)+p(x, y)$,

where || || represents the Euclidean distance from the current point to the center of the radial function. 
In this article, the approach proposed in Ref. [54] was used to estimate the deformation. This technique uses the thin plate radial function with affine transforms to represent the deformation in a pair of $2 \mathrm{D}$ surfaces and can be expressed as follows:

$$
\begin{gathered}
f_{x}(x, y)=\sum_{i} \quad c_{i} S\left(\sqrt{\left(x-x_{i}\right)^{2}+\left(y-y_{i}\right)^{2}}\right)+a_{0}+a_{1} \times x+a_{2} \times y=x^{\prime} \\
f_{y}(x, y)=\sum_{i} \quad c_{i} S\left(\sqrt{\left(x-x_{i}\right)^{2}+\left(y-y_{i}\right)^{2}}\right)+b_{0}+b_{1} \times x+b_{2} \times y=y^{\prime} \\
S(t)=t^{2} \times \log \left(t^{2}\right)
\end{gathered}
$$

Therefore, to find out the deformation for a given point $(x, y)$ using a set of $n$ anchor points $\left(x_{i}, y_{i}\right)$ where the position $\left(x_{i}{ }^{\prime}, y_{i}{ }^{\prime}\right)$ after deformation is known, the coefficients of the model can be calculated as the solution of a system with $(n+3)$ simultaneous linear equations as proposed in Ref. [54].

\section{Results and discussion}

To validate the proposed technique, the conducted experiments were performed calculating the error statistics of every technique according to the ground truth data [55]. The endpoint (EP) error metric defined in Eq. (10) was used to measure the accuracy of the techniques:

$E P=\sqrt{\left(u_{M}-u_{T}\right)^{2}+\left(v_{M}-v_{T}\right)^{2}}$,

where $\left(u_{M}, v_{M}\right)$ is the measured flow in a point and $\left(u_{T}, v_{T}\right)$ is the true flow. The EP error represents the Euclidian distance with the real displacement, and it is more relevant than the angular error (AE) [55].

The results obtained with the proposed algorithm were compared with the following state-ofthe-art techniques:

- The block-matching technique provided by the computer vision library OPENCV available in Ref. [56].

- Modern implementations of the classic Black and Anandan technique and Horn and Schunck technique carried out in Ref. [57]. These implementations can be found in Ref. [58].

- The classic + Non Local term technique carried out in Ref. [59] and available in Ref. [58]

- A variational optical flow technique carried out in Ref. [60], extended in Ref. [61], and available in Ref. [62].

The proposed technique was used to analyze the proteins of interest using $55 \times 55$ blocks built around the proteins. Three iterations were used, including two levels of pyramidal decomposition with homography deformation models and using the radial basis function deformation model in the last iteration.

An example of the output obtained with the proposed technique is shown in Fig. 4. The use of deformation models allows obtaining the full displacement field from the selected proteins and obtaining a registered image warping the test image to match the reference image. Fig. 5 shows the accuracy of the obtained registered image. 

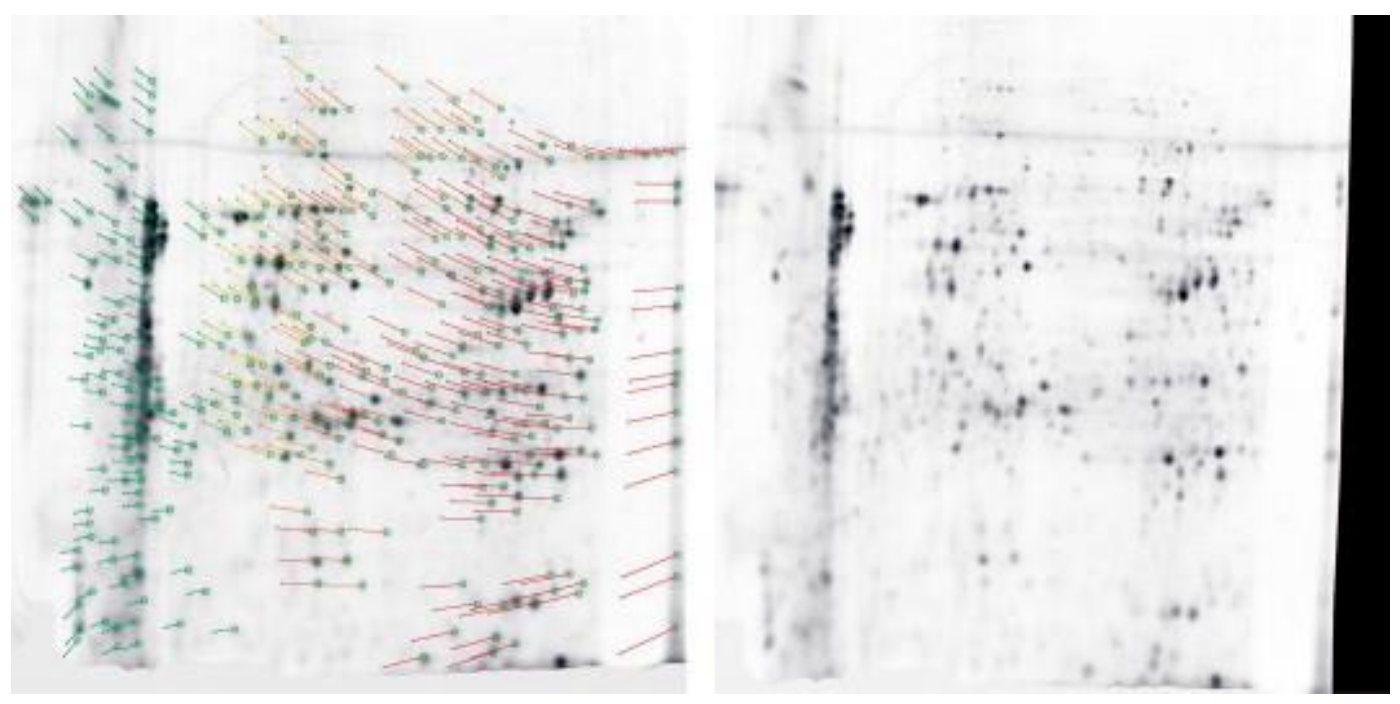

Fig.4. Results obtained with the proposed technique for the reference and test images from Fig. 1. Left: Matching of proteins represented as vectors superimposed to the test image. Right: Registered test image representing the test image warped to match the reference image.
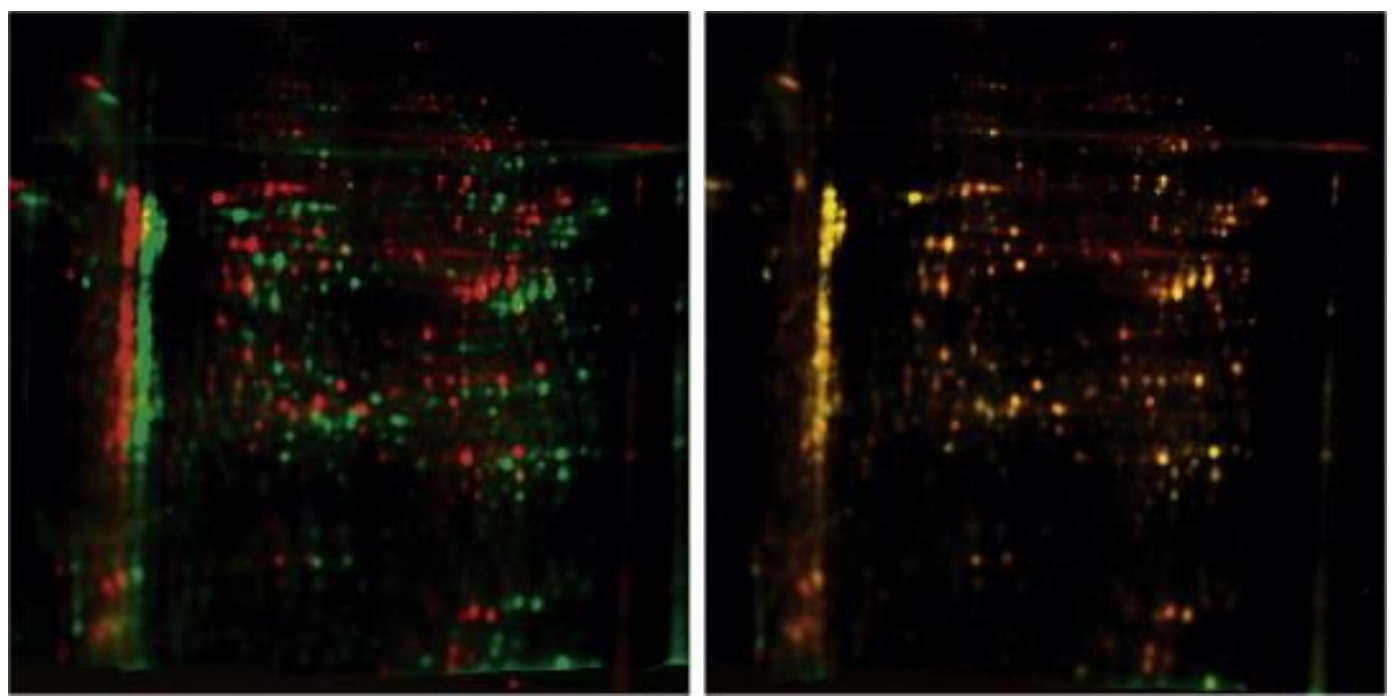

Fig.5. Accuracy of registering the example of Fig. 1. Left: Reference image, colored in red, superimposed to the original test image, colored in green. Right: Reference image, colored in red, superimposed to the registered image, colored in green. Overlapped spots can be seen in yellow. (For interpretation of the references to color in this figure legend, the reader is referred to the Web version of this article.)

The configuration used for the other block-matching techniques was as similar as possible to the one used with the proposed technique. The configurations for the rest of the techniques were those suggested by their respective authors, using the default parameters (which are supposedly optimal to the Middlebury training set) whenever possible [63].

In analyzing the obtained results (Table 1), it can be seen that the proposed technique produced significantly more accurate results than all of the other techniques in the gel scenario. In addition, the execution time was significantly reduced because it was not necessary to analyze the full image. 
Table 1. Comparative results from the test with $2 \mathrm{D}$ gel images.

\begin{tabular}{lccccc}
\hline Accuracy & Average error (pixel) & SD error (pixel) & Correct (\%) & Missed (\%) & Execution time (s) \\
\hline & & & & & 1027.5 \\
BA & 18.65 & 39.74 & 84.0 & 16.0 & 297.5 \\
HS & 21.36 & 47.33 & 83.8 & 16.2 & 2326.5 \\
Classic + NL & 34.88 & 62.87 & 69.5 & 30.5 & 77.8 \\
Brox & 24.36 & 19.44 & 34.6 & 65.4 & 43117.5 \\
OPENCV & 34.85 & 39.15 & 55.2 & 44.8 & 22.3 \\
Our technique & 3.10 & 4.94 & 96.6 & 3.4 & \\
\end{tabular}

Note: SD, standard deviation; BA, Black and Anandan; HS, Horn and Schunck; OPENCV, Open Source Computer Vision; NL, Non Local term.

\section{Conclusions}

The current article has proposed a new iterative block-matching technique to solve the problem of protein matching in 2D-PAGE images. The proposed algorithm can be used with different deformation models with different degrees of freedom that can be imposed in different stages of the algorithm, so the measurements can be obtained by using a regular grid of blocks and a local deformation model or by analyzing an arbitrary set of points and a global model.

The proposed technique outperformed others in the 2D electrophoresis gels field, improving the results obtained by different state-of-the-art algorithms.

Finally, a new methodology to conduct and evaluate experiments with $2 \mathrm{D}$ electrophoresis gels has been carried out.

\section{Acknowledgments}

This work was partially supported by the General Directorate of Research, Development, and Innovation (Dirección Xeral de Investigación, Desenvolvemento e Innovación) of the Xunta de Galicia (10MDS014CT) by the General Directorate of Culture, Education, and University Management of the Xunta de Galicia (10SIN105004PR). It was partially funded by the Carlos III Health Institute (Collaborative Project on Medical Informatics, PI13/00280).

\section{References}

[1]. T. Rabilloud, M. Chevallet, S. Luche, C. Lelong. Two-dimensional gel electrophoresis in proteomics: past, present, and future. J. Proteomics, 73 (2010), pp. 2064-2077.

[2]. A.G.M. Almansa, A. Pardo, J. Preciozzi, Processing of 2D electrophoresis gels, in: Presented at the 1st International Workshop on Computer Vision Applications for Developing Regions, 2007.

[3]. T. Voss, P. Haberl. Observations on the reproducibility and matching efficiency of two-dimensional electrophoresis gels: consequences for comprehensive data analysis. Electrophoresis, 21 (2000), pp. $3345-3350$.

[4]. S. Veeser, M.J. Dunn, G.-Z. Yang. Multiresolution image registration for two-dimensional gel electrophoresis. Proteomics, 1 (2001), pp. 856-870.

[5]. B. Raman, A. Cheung, M.R. Marten. Quantitative comparison and evaluation of two commercially available, two-dimensional electrophoresis image analysis software packages, Z3 and Melanie. Electrophoresis, 23 (2002), pp. 2194-2202.

[6]. A.W. Dowsey, M.J. Dunn, G.-Z. Yang. Automated image alignment for 2D gel electrophoresis in a highthroughput proteomics pipeline. Bioinformatics, 24 (2008), pp. 950-957. 
[7]. A. Fusiello, V. Roberto. Symmetric stereo with multiple windowing. Int. J. Pattern Recognit. Artificial Intell., 14 (2000), pp. 1053-1066.

[8]. M. Raffel, C. Willert, J. Kompenhans. Particle Image Velocimetry: A Practical Guide. Springer, New York (2000)

[9]. D. Schwarz, T. Kasparek. Multilevel block matching technique with the use of generalized partial interpolation for nonlinear intersubject registration of MRI brain images. Eur. J. Biomed. Informatics, 1 (2006), pp. 90-97.

[10]. T. Amiaz, E. Lubetzky, M. Kiryati. Coarse to over-fine optical flow estimation. Pattern Recognit., 40 (2007), pp. 2496-2503.

[11]. J. Liangbao, C. Jiao, C. Xuehong, C. Rui. Hierarchical support-weight block matching approach in depth extraction. Int. J. Digital Content Technol. Appl., 5 (2011), pp. 30-38.

[12]. H.-S. Oh, H.-K. Lee. Block matching algorithm based on an adaptative reduction of the search area for motion estimation. Real Time Imaging, 6 (2000), pp. 407-414.

[13]. Y. Luo, M. Celenk, A hybrid block-matching approach to motion estimation with adaptive search area, in: Presented at the 15th International Conference on Systems, Signals, and Image Processing (IWSSIP), 2008.

[14]. D. Aboutajdine, F. Essannouni, Fast block matching algorithms using frequency domain, in: Presented at the International Conference on Multimedia Computing and Systems (ICMCS), 2011

[15]. B.D. Lucas, T. Kanade, An iterative image registration technique with an application to stereo vision, in: Presented at the Imaging Understanding Workshop, 1981.

[16]. M. Raffel, C. Willert, J. Kompenhans. Particle Image Velocimetry: A Practical Guide. (2nd ed.)Springer, New York (2007).

[17]. A. Rodriguez, C. Fernandez-Lozano, J.-A. Seoane, J. Rabuñal, J. Dorado, Motion estimation in real deformation processes based on block-matching techniques, in: Presented at the IEEE International Symposium on Signal Processing and Information Technology (ISSPIT), 2011.

[18]. U. Malsch, C. Thieke, P.E. Huber, R. Bendl. An enhanced block matching algorithm for fast elastic registration in adaptive radiotherapy. Phys. Med. Biol., 51 (2006), pp. 4789-4806.

[19]. X. Yuan, X. Shen, Block matching algorithm based on particle swarm optimization for motion estimation, in: Presented at the International Conference on Embedded Software and Systems (ICESS), 2008.

[20]. D. Zhāng, G. Lu, An edge and color oriented optical flow estimation using block matching, in: Presented at the International Conference on Signal Processing (ICSP), 2000.

[21]. X. Shi, J. Chen, Image mosaics algorithm based on feature-block matching, in: Presented at the International Conference on Multimedia Technology (ICMT), 2011.

[22]. M.A. Sekeh, M.A. Maarof, M.F. Rohani, M. Motiei, Efficient image block matching algorithm with two layer feature extraction, in: 7th International Conference on Information Technology in Asia (CITA), 2011, pp. 1-5.

[23]. I. Stuke, T. Aach, E. Barth, C. Mota. Multiple motion estimation by block-matching using MRF. Int. J. Comput. Inf. Sci., 5 (1) (2004).

[24]. X. Ren, Local grouping for optical flow, in; Presented at the IEEE Computer Society Conference on Computer Vision and Pattern Recognition (CVPR), 2008.

[25]. C. Rhemann, A. Hosni, C. Rother, M. Gelautz, Fast cost-volume filtering for visual correspondence and beyond, in: Presented at the IEEE Computer Society Conference on Computer Vision and Pattern Recognition (CVPR), 2011.

[26]. S.X. Ju, M.J. Black, A.D. Jepson, Skin and bones: multi-layer, locally affine, optical flow, and regularization with transparency, in: Presented at the IEEE Conference on Computer Vision and Pattern Recognition (CVPR), 1996.

[27]. M.H. Chan, Y.B. Yu, A.G. Constantinides, Variable size block matching motion compensation with applications to video coding, in: IEE Proceedings I: Communications, Speech, and Vision, 1990, pp. 205212.

[28]. T. Kanade, M. Okutomi. A stereo matching algorithm with an adaptive window: theory and experiment. IEEE Trans. Pattern Anal. Mach. Intell., 16 (1994), pp. 920-932.

[29]. G. R. Martin, R. A. Packwood, I. Rhee, Variable size block matching motion estimation with minimal error, in: Presented at SPIE Digital Video Compression: Algorithms and Technologies, 1996.

[30]. S.B. Kang, R. Szeliski, J. Chai, Handling occlusions in dense multi-view stereo, in: IEEE Conference on Computer Vision and Pattern Recognition (CVPR), vol. 1, 2001, pp. 103-110.

[31]. O. Veksler, Fast variable window for stereo correspondence using integral images, in: IEEE Conference on Computer Vision and Pattern Recognition (CVPR), vol. 1, 2003, pp. 556-561.

[32]. M. Gong, R. Yang, L. Wang, M. Gong. A performance study on different cost aggregation approaches used in real-time stereo matching. Int. J. Comput. Vision, 75 (2007), pp. 283-296.

[33]. K.-J. Yoon, S. Kweon. Adaptive support-weight approach for correspondence search. IEEE Trans. Pattern Anal. Mach. Intell., 24 (2006), pp. 650-656.

[34]. J. Delon, B. Rougé. Small baseline stereovision. J. Math. Imaging Vision, 28 (2007), pp. 209-223. 
[35]. C. Schmid, A. Zisserman. The geometry and matching of lines and curves over multiple views. Int. J. Comput. Vision, 40 (2000), pp. 199-234.

[36]. A. Rodriguez, J. Rabuñal, J.L. Perez, F. Martinez-Abella. Optical analysis of strength tests based on block-matching techniques. Comput. Aided Civil Infrastruct. Eng., 27 (2012), pp. 573-593.

[37]. S. Ourselin, A. Roche, S. Prima, N. Ayache. Block matching: a general framework to improve robustness of rigid registration of medical images. Lecture Notes Comput. Sci., 2000 (1935), pp. $557-$ 566.

[38]. N. Wiest-Daessle, S. Prima, Block-matching strategies for rigid registration of multimodal medical images, in: Presented at the IEEE International Symposium on Biomedical Imaging (ISBI), 2012.

[39]. A. Basarab, W. Aoudi, H. Liebgott, D. Vray, P. Delachartre, Parametric deformable block matching for ultrasound imaging, in: Presented at the IEEE International Conference on Image Processing, 2007.

[40]. J.K. Dave, F. Forsberg, S. Fernandes, C.W. Piccoli, T.B. Fox, D.A. Merton, L.M. Leodore, A.L. Hall. Static and dynamic cumulative maximum intensity display mode for subharmonic breast imaging: a comparative study with mammographic and conventional ultrasound techniques. J. Ultrasound Med., 29 (2010), pp. 1177-1185.

[41]. M.G. Linguraru, N.V. Vasilyev, G.R. Marx, W. Tworetzky, P.J. Tworetzky, R.D. Howe. Fast block tracking of trial septal defects in 4D echocardiography. Med. Image Anal., 2008 (2008), pp. 349-412.

[42]. S.T. Nevo, M. van Stralen, A.M. Vossepoel, J.H. Reiber, N. de Jong, A.F. van der Steen, J.G. Bosch Automated tracking of the mitral valve annulus motion in apical echocardiographic images using multidimensional dynamic programming. Ultrasound Med. Biol., 33 (2007), pp. 1389-1399.

[43]. E. Nielsen, F. Orderud, H. G. Torp, Performance optimization of block matching in 3D echocardiography, in: Presented at the IEEE International Ultrasonics Symposium (IUS), 2009.

[44]. O. Clatz, H. Delingette, I.-F. Talos, A.J. Golby, R. Kikinis, F.A. Jolesz, N. Ayache, S.K. Warfield. Robust nonrigid registration to capture brain shift from intraoperative MRI. IEEE Trans. Med. Imaging, 24 (2005), pp. 1417-1427.

[45]. D. Schwarz, T. Kasparek, Multilevel block matching technique with the use of generalized partial volume interpolation for nonlinear intersubject registration of MRI brain images, in: Presented at the European Signal Processing Conference (EUSIPCO), 2008.

[46]. K. Rohr, P. Cathier, S. Wörz. Elastic registration of electrophoresis images using intensity information and point landmarks. Pattern Recognit., 37 (2004), pp. 1035-1048.

[47]. S. Wörz, M.L. Winz, K. Rohr, Geometric alignment of 2D gel electrophoresis images using physicsbased elastic registration, in: Presented at the IEEE International Symposium on Biomedical Imaging: From Nano to Macro, 2008.

[48]. J. Weekes, C.H. Wheeler, J.X. Yan, J. Weil, T. Eschenhagen, G. Scholtysik, M.J. Dunn. Bovine dilated cardiomyopathy: proteomic analysis of an animal model of human dilated cardiomyopathy. Electrophoresis, 20 (1998), pp. 898-906.

[49]. M.Y. Heinke, C.H. Wheeler, J.X. Yan, V. Amin, D. Chang, R. Einstein, M.J. Dunn, C.G. dos Remedios. Changes in myocardial protein expression in pacing-induced canine heart failure. Electrophoresis, 20 (1999), pp. 2086-2093.

[50]. O. Langella, M. Zivy. A method based on bead flows for spot detection on 2-D gel images. Proteomics, 8 (2008), pp. 4914-4918.

[51]. B. Karaçali. Information theoretic deformable registration using local image information. Int. J. Comput. Vision, 72 (2007), pp. 219-237.

[52]. C.A. Glasbey, K.V. Mardia. A review of image warping methods. J. Appl. Statist., 25 (1998), pp. 155171.

[53]. N. Arad, N. Dyn, D. Reisfeld, Y. Yeshurun. Image warping by radial basis functions: application to facial expressions. Graph. Models Image Process., 1 (1994), pp. 161-172.

[54]. F.L. Bookstein. Principal warps: thin-plate splines and the decomposition of deformations. IEEE Trans. Pattern Anal. Mach. Intell., 11 (1989), pp. 567-585.

[55]. S. Baker, D. Scharstein, J.P. Lewis. A database and evaluation methodology for optical flow. Int. Conf. Comput. Vision, 92 (2007), pp. 1-31.

[56]. Open Source Computer Vision, Open Source Computer Vision (OPENCV), 2012/2013, http://opencv.org.

[57]. D. Sun, S. Roth, J.P. Lewis, M.J. Black, Learning optical flow, in: Presented at the European Conference on Computer Vision (ECCV), 2008.

[58]. D. Sun, Dewing Sun research page, Computer Science Department, Brown University, 2012/2013, http://www.cs.brown.edu/ dqsun..

[59]. D. Sun, S. Roth, M.J. Black, Secrets of optical flow estimation and their principles, in: Presented at the IEEE Computer Society Conference on Computer Vision and Pattern Recognition (CVPR), 2010.

[60]. T. Brox, A. Bruhn, N. Papenberg, J. Weickert, High accuracy optical flow estimation based on a theory for warping, in: Presented at the European Conference on Computer Vision (ECCV), 2004.

[61]. N. Papenberg, A. Bruhn, T. Brox, S. Didas, J. Weickert. Highly accurate optic flow computation with theoretically justified warping. Int. J. Comput. Vision, 67 (2006), pp. 141-158. 
[62]. Freiburg Computer Vision Group, Binaries/Code, 2012/2013, http://lmb.informatik.unifreiburg.de/resources/binaries.

[63]. Middlebury, The Middlebury Computer Vision Pages, 2012/2013, http://vision.middlebury.edu. 